\title{
Primary Intramedullary Meningeal Melanocytoma in Cervical Spine: A Case Report and Literature Review
}

\author{
Keonhee $\mathrm{Kim}^{1}$, Jaeyon $\mathrm{Choi}^{2}$, Yoon Jin $\mathrm{Cha}^{3}$, Kyung Hyun $\mathrm{Kim}^{4}$, Yong Eun $\mathrm{Cho}^{4}$ \\ ${ }^{1}$ Department of Neurosurgery, Severance Hospital, Yonsei University College of Medicine, Seoul, Republic of Korea \\ ${ }^{2}$ Department of Neurosurgery, Seoul Hospital, Ehwa University Medical Center, Seoul, Republic of Korea \\ ${ }^{3}$ Department of Pathology, Gangnam Severance Hospital, Yonsei University College of Medicine, Seoul, Republic of Korea \\ ${ }^{4}$ Department of Neurosurgery, Spine and Spinal Cord Institute, Gangnam Severance Hospital, Yonsei University College of Medicine, \\ Seoul, Republic of Korea
}

Corresponding author: Yong Eun Cho, MD, PhD

Department of Neurosurgery, Spine and Spinal Cord Institute, Gangnam Severance Hospital, 211 Eonju-ro, Gangnam-gu, Seoul, 06273, Republic of Korea

Tel: $+82-2-2072-2358$

Fax: $+82-2-744-8459$

E-mail: ydnscho@gmail.com

Received: October 29, 2020

Revised: December 10, 2020

Accepted: December 23, 2020
Primary intramedullary meningeal melanocytoma is an extremely rare tumor that occurs in the spinal cord. To our knowledge, 29 cases have been reported. We report a case of intramedullary meningeal melanocytoma with a narrative literature review.: A 78-year-old man with the chief complaint of rightside weakness over a course of six months. Magnetic resonance imaging revealed an intradural intramedullary tumor between C3 and C5 levels that showed hypo-intensity on T2 and mild homogenous hyper-intensity on gadolinium-enhanced T1-weighted images. Myelotomy was performed for tumor removal, and a black-pigmented tumor was observed under surgical microscope. Histopathological examination confirmed intramedullary meningeal melanocytoma. Since radiologic findings are insufficient to diagnose primary intramedullary meningeal melanocytoma, histopathological exami nation must be performed for diagnosis. Complete tumor resection is the most important factor of prognosis, and adjuvant radiotherapy should be considered to prevent malignant transformation and recurrence.

Key Words: Meningeal melanocytoma, Intramedullary melanocytoma, CNS tumor, Oncology

\section{INTRODUCTION}

Intramedullary meningeal melanocytoma is an exceptionally rare tumor that occurs in the central nervous system. Meningeal melanocytoma was first described in 1972 by Limas and Tio, defined as a pigmented tumor of the leptomeninges'. Intramedullary meningeal melanocytoma is known to be derived from melanocytes located in leptomeninges ${ }^{2,3}$. During the embryologic period, melanocytes travel from leptomeninges to the spine, and cellular proliferation affects the characteristics of the cell to become a tumor. From the database of PubMed and Google Scholar, a total of 29 cases of intramedullary meningeal melanocytoma were found between 1970 and 2020. Here, we report a case of intramedullary menin gealmelanocytoma in the spinal cord and provide an overview of the available literature. Additionally, this is the first case report of the same in Korea.

\section{CASE REPORT}

A 78-year-old man was admitted to our institution with the chief complaint of severe low back pain for 30 years and progressive right-side weakness over a course of six months. No remarkable past medico-surgical history was found. On physical examination, the patient suffered from low back pain around the area of $L 2$ to L4. Mild direct tenderness was observed. On neurologic examination, the straight leg raising test was positive for both legs: $30^{\circ}$ and $70^{\circ}$ for the right and left legs, respectively. There was no posterior neck pain or direct tendemess on the neck, but the patient occasionally dropped an object against his will. In addition, gait disturbance was observed. The right-side motor weakness was present, and the general motor grade was between II and III on the right side. The patient suffe red from right-sided numbness.

No remarkable abnormalities were found in the plane cenvical $X$-ray images, except for degenerative disc space narrowing lesions. Computed tomography $(\mathrm{CT})$ images revealed a hyperdense mass between C3 and C5 levels. The center of mass was heterogeneous. Spinal cord tumor was suspected from the images.

Magnetic resonance imaging (MRI) of the cervical and lumbar spine showed cervical intradural-intramedullary tumor between C3 and $\mathrm{C} 5$ levels and epidural abscess between $\mathrm{L} 3$ and $\mathrm{S} 1$ levels. The cervical lesion showed hypo-intensity on T2 and hyper-intensity on T1-weighted images (TIM). Diffuse mild enhancement was revealed in gadolinium-enhanced T1-weighted images (Figure 1). MRI also showed a $21 \mathrm{~mm}$ mass with hemorhagic changes containing multiple septa in the center. The mass was skewed toward the right from the center of the spine. Based on T2-weighted images, it was suspected to be edematous and contained high vascularity. The initial impressions based on the cervical MRI findings were cavernous malformation, arteriovenous malformation, meningioma, and lymphoma. 
Abnormal enhancement was observed on lumbar MRI in the vertebrae, intervertebral discs, and ventral epidural space between L4 and S1 levels (Figure 2). The results suggested infectious spondylodiscitis and multiple infected cysts in the right facet joints between L4 and S1 levels. The infection was confirmed comprehensively, and ceftriaxone and vancomycin were introduced to the patient for conservative treatment.

Surgical intervention was performed for the cenvical lesion. During the operation, laminectomy was performed between $\mathrm{C} 3$ and $\mathrm{C} 5$ levels. After opening the dura mater under a surgical microscope, a normal
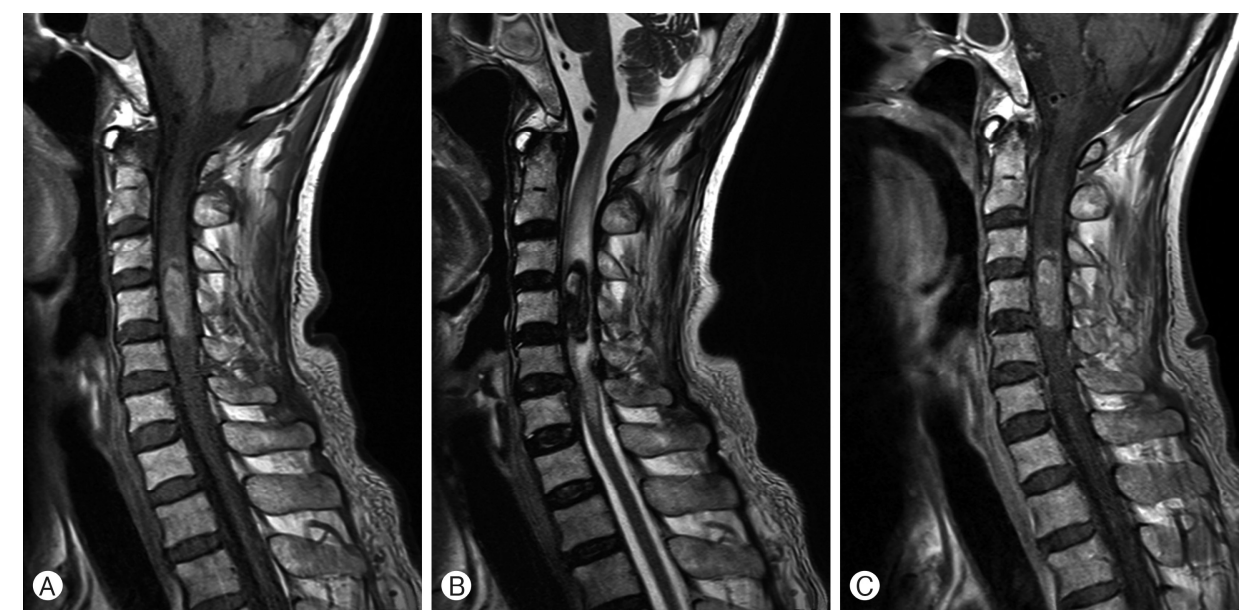

Figure 1. Sagittal image of magnetic resonance imaging between $\mathrm{C} 3$ and $\mathrm{C} 5$ levels before the operation. (A): T1-weighted images slightly show hyperintensity. (B): Hypointensed lesion can be observed in T2-weight images with multiple septa in center. (C): Gadolinium enhanced the mass slightly, which is revealed in the enhanced $\mathrm{Tl}$-weighted images.
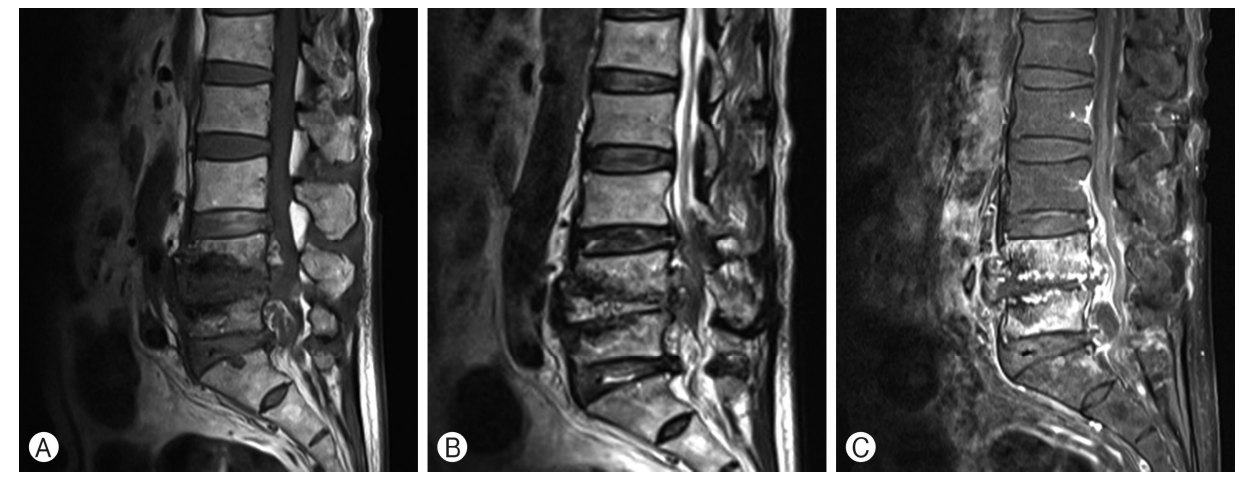

Figure 2. Sagittal image of magnetic resonance imaging between L1 and L5 levels. (A): T1-weighted images show hypointensity on L4\&5, \&S1 vertebral body and intervertebral disc spaces. (B): Heterogenous hypointensed lesions can be observed in T2-weight images. (C): Gadolinium enhanced the lesion, which is revealed in the enhanced $\mathrm{Tl}$-weighted images.


Figure 3. Intramedullary meningeal melanocytoma images under surgical microscope. (A): There is no sign of pigmented tumor in the subdural space. (B): Solid and vascularized mass can be seen located in the intramedullary space. (C): The pigmented mass, completely removed from the spinal cord. 
colored spinal cord was noted. The surface of the right spinal cord felt harder than the left spinal cord at the C4 level. After opening the pia mater and meticulous spinal cord dissection in the midline, a black pigmented tumor mass was exposed (Figure 3). The mass had a rubbery hard consistency and was surrounded with soft vascularized tissue. However, there was no hemorrhagic evidence, such as old blood clots. The demarcation of the fumor from the normal spinal cord was good in color, but it was not easy to find a cleavage plane between the tumor mass and surrounding normal spinal cord parenchyma. With meticulous dissection using dissector and intemal decompression with an ultrasonic aspirator, the tumor was completely removed (Figure 3). During the procedure, intraoperative neuromonitoring was performed. Initially, motor evoked potentials (MEP) showed a $70 \%$ decrease in the right upper extremity compared to the left. During the procedure of tumor removal, the MEP wave
(A)
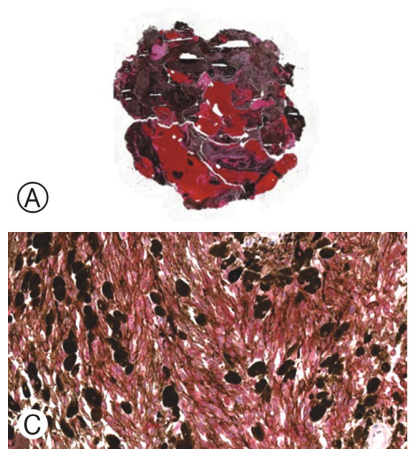
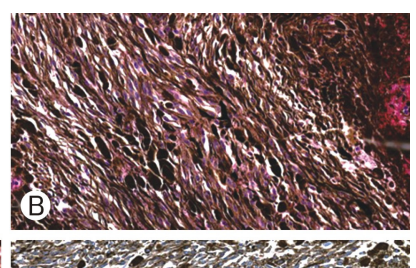

Figure 4. (A): Hematoxylin \& Eosin (H\&E) stain, 5-folded magnification; scan power view shows black pigmented lesion associated with hemorrhage. (B): H\&E stain, 30-folded magnification; on high power magnification, pigmented spindle tumor cells and heavily pigmented macrophages can be seen. Note that the oval nuclei of tumor cells lack cytological atypia. (C): Melan-A stain; tumor cells were positive for melan-A immunohistochemistry. (D): S-100 stain; tumor cells were negative for S-100 immunohistochemistry. changed to a flat condition and remained the same by the end of surgery.

Immediately after the operation, the right-side general motor was recorded to be grade $I$. The patient suffered from post-operative paralytic ileus. A Levin tube was inserted to decrease the abdominal pressure. Progressively, the patient recovered. Antibiotics were retained during the admission period. However, the right-sided hemiparesis did not show any improvement.

Microscopic analysis was performed for pathological diagnosis. Morphologically, there was a little activity of mitosis and no severe dysplasia. The cells were immunochemically stained with Melan-A and S-100 proteins. CD-68 was positive, and the percentage of $\mathrm{Ki}$ 67 was less than $5 \%$. The official result was a benign tumor that was suitable for melanocytoma (Figure 4).

A proton emission tomography (PET) scan was performed to search for a primary malignant lesion. Only mild F-18 fluorodeoxyglucose (FDG) uptake was observed in the spinal cord between C3 and C5 levels without a clearly defined margin, which indicates low metabolic tumor or focal inflammatory process. There was no sign of distant metastasis. MRI was performed on the second day of the operation, and it confirmed the complete resection of the tumor (Figure 5).

\section{DISCUSSION}

\section{Characteristics}

The authors described the tumor as solitary and low-grade neoplasm with the characteristic of invading surrounding structures'. In general, meningeal melanocytoma is usually located in the intradural extramedullary compartment and is adhesive to the meninges. Intramedullary meningeal melanocytoma in the spinal cord is known to be an extremely rare tumor. The previously reported cases are summanized in Table 1. There is no large volume of analyzed research due to its low incidence rate. However, it can be presumed from the previously reported cases that melanocytoma of the spinal cord occurs
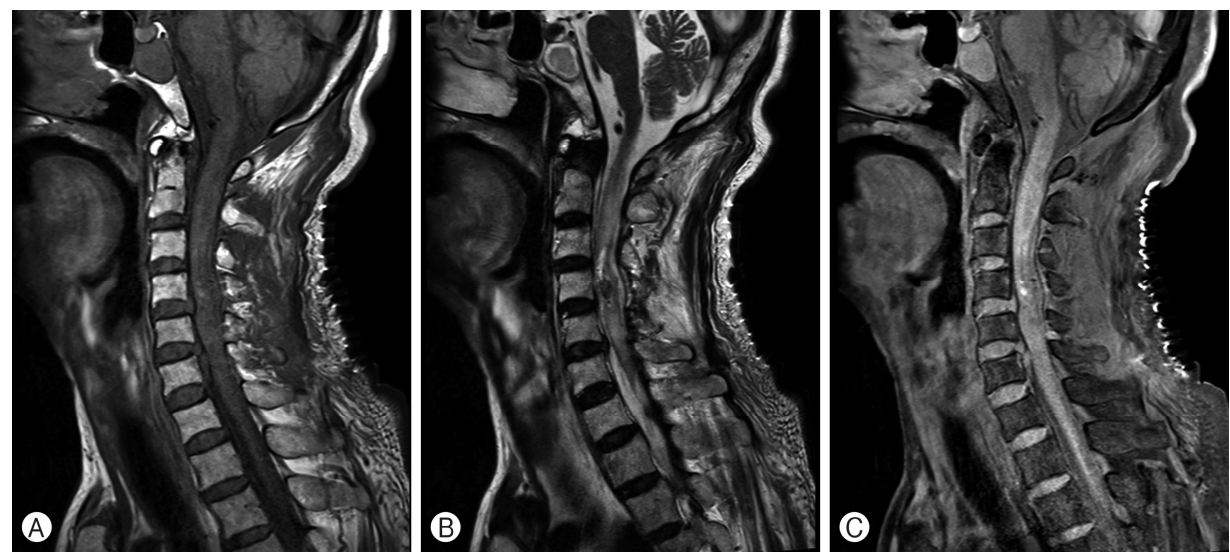

Figure 5. Sagittal image of magnetic resonance imaging between C3 and C5 levels after the operation. (A): T1-weighted images show no sign of remaining mass on the cervical levels. (B): The hypointensed lesion revealed in the pre-operative images disappeared after the surgery. (C): No sign of definitely enhanced mass in the cervical levels. 
Table 1. Summary of previously reported intramedullary melanocytoma cases

\begin{tabular}{|c|c|c|c|c|c|c|c|c|c|}
\hline \multicolumn{2}{|l|}{ Authors/Reported year } & \multirow{2}{*}{$\begin{array}{c}\text { Age } \\
49\end{array}$} & \multirow{2}{*}{\begin{tabular}{|l|} 
Sex \\
Female
\end{tabular}} & \multirow{2}{*}{\begin{tabular}{|l|} 
Location \\
Thoracic
\end{tabular}} & \multirow{2}{*}{\begin{tabular}{|l|} 
Location \\
T10-12 \\
\end{tabular}} & \multirow{2}{*}{\begin{tabular}{|l} 
Resection \\
Subtotal
\end{tabular}} & \multirow{2}{*}{$\begin{array}{l}\text { RTx } \\
\text { No }\end{array}$} & \multirow{2}{*}{$\begin{array}{c}\text { Recur. } \\
\text { Yes }\end{array}$} & \multirow{2}{*}{$\begin{array}{r}\mathrm{FU} \\
48\end{array}$} \\
\hline Barth et al. $^{18}$ & 1993 & & & & & & & & \\
\hline Glick et al. $^{19}$ & 1997 & 69 & Male & Cervical & $\mathrm{Cl}-2$ & Total & No & No & 60 \\
\hline & & 39 & Female & Thoracic & T8-9 & Subtotal & Yes & No & 12 \\
\hline & & 27 & Female & Thoracic & T1-6 & Total & No & No & 24 \\
\hline & & 56 & Female & Thoracic & T12 & Total & No & No & 48 \\
\hline & & 74 & Female & Thoracic & T11-12 & Total & No & No & 12 \\
\hline & & 70 & Male & Cenvical & $\mathrm{Cl}$ & Total & - & - & - \\
\hline & & 24 & Female & Thoracolumbar & T12-L1 & Total & No & No & 24 \\
\hline Rades et al. ${ }^{16}$ & 2001 & 23 & Female & Thoracic & $\mathrm{T} 4-7$ & Subtotal & Yes & Yes & 54 \\
\hline Delhaye et al. ${ }^{20}$ & 2001 & 38 & Female & Thoracic & T6-T9 & Subtotal & No & Yes & 48 \\
\hline lida et al. $^{21}$ & 2002 & 42 & Male & Thoracic & $\mathrm{T10}$ & $n / a$ & No & No & 4 \\
\hline Turhan et al. ${ }^{22}$ & 2004 & 19 & Female & Thoracic & T8 & Total & No & No & 36 \\
\hline Van Paesscehn et al. ${ }^{23}$ & 2004 & 51 & Male & Cervical & $\mathrm{Cl}-2$ & Total & - & - & - \\
\hline Horn et al. ${ }^{24}$ & 2008 & 37 & Female & Cervical & $\mathrm{C} 1-3$ & Total & No & Yes & 38 \\
\hline & & 37 & Female & Thoracic & T9-10 & Total & No & Yes & 16 \\
\hline & & 48 & Male & Thoracic & $\mathrm{T} 12$ & Total & No & Yes & 185 \\
\hline Chacko et al. ${ }^{25}$ & 2008 & 22 & Male & Thoracic & T6-11 & Total & No & No & 96 \\
\hline Karikari et al. ${ }^{10}$ & 2009 & 32 & Female & Thoracic & T10 & Total & No & No & 3 \\
\hline & & 20 & Male & Thoracic & T12 & Total & No & No & 2 \\
\hline Caruso et al. ${ }^{26}$ & 2009 & 62 & Male & Thoracic & T1 1-12 & Total & No & No & 24 \\
\hline Perrini et al. ${ }^{27}$ & 2009 & 79 & Female & Thoracic & T10-11 & Subtotal & Yes & No & 30 \\
\hline Eskandari et al. ${ }^{28}$ & 2010 & 45 & Male & Thoracic & T11 & Subtotal & Yes & Yes & 36 \\
\hline Muthappan et al. ${ }^{29}$ & 2012 & 61 & Female & Cenvical & C3-4 & Total & No & No & 36 \\
\hline Kahilogullari ${ }^{30}$ & 2012 & 28 & Female & Thoracic & - & Total & - & - & - \\
\hline Wagner $^{31}$ & 2015 & 63 & Male & Cervical & $\mathrm{C} 2-3$ & Total & Yes & Yes & 18 \\
\hline Reutov $^{32}$ & 2016 & 28 & Female & Cervical & $\mathrm{C} 2-3$ & Subtotal & No & No & 24 \\
\hline Khoulal et al. ${ }^{33}$ & 2018 & 51 & Female & Cervicothoracic & C7-T1 & Subtotal & Yes & - & - \\
\hline Dubey et al. ${ }^{9}$ & 2018 & 35 & Female & Cervicothoracic & C6-T6 & Subtotal & No & No & 6 \\
\hline Hoffmann et al. ${ }^{15}$ & 2016 & 58 & Female & Thoracic & T9-10 & Total & Yes & No & 3 \\
\hline Present case & 2020 & 78 & Male & Cenvical & C3-5 & Total & & & \\
\hline
\end{tabular}

*RTx: Radiotherapy, Recur.: recurrence, FR: Followup (months)

most often in the thoracic levels $(n=19)$ (Table 2).

\section{Embryology}

The epidermal melanoblasts reach leptomeninges during the embryogenic period of formation of the neural crest $^{4}$. The neural crest undergoes differentiation to become numerous cell types, including the leptomeninges, glial cells, adrenal medullary cells, and melanoctyes ${ }^{5}$. The lateral margin of the thin layer from the neural crest forms the leptomeninges after embryogenesis. Melanocytes become atypical cells that move toward malignancy with cellular differentiation and proliferation. In vitro experiments have revealed that melanocyte cells become immature ancestral cells after exposure to high concentrations of endothelin-3 substance ${ }^{6}$. In vivo, this phenomenon might be a clue toward understanding the pathogenesis
Table 2. Location, Sex, Age at diagnose from the reported cases

\begin{tabular}{|c|c|c|c|c|c|}
\hline Location & Cenvical & $\begin{array}{l}\text { Cervico- } \\
\text { thoracic }\end{array}$ & Thoracic & $\begin{array}{c}\text { Thoraco- } \\
\text { lumbar }\end{array}$ & Lumbar \\
\hline \multirow{3}{*}{ Sex } & $8(26.7 \%)$ & 2(6.7\%) & 19(63.3\%) & $1(3 \%)$ & 0 \\
\hline & Female & Male & & & \\
\hline & & 11 & & & \\
\hline \multirow[t]{2}{*}{$\begin{array}{l}\text { Age at } \\
\text { Diagnose }\end{array}$} & $10-30$ & $30-50$ & & & \\
\hline & 8 & 11 & 11 & & \\
\hline
\end{tabular}

of melanocyte ${ }^{7}$. Additionally, the tumor suppressor genes and proteins, CDKN2A, pl6 $6^{\text {INA }}$, p19 $9^{\text {ARF }}$, and PTEN, play a critical role in transformation ${ }^{8}$.

Melanocytoma is classified according to the World Health Organi- 
zation classification under the subclass of melanocytic lesions of the CNS. The subclass includes other melanocytic tumors such as melancytosis, melanomatosis, and malignant melanoma?. Most cases of meningeal melanocytoma within the spinal cord reported in the literature have been located in the intradural extramedullary compartment ${ }^{10}$. Incidence of intramedullary melanocytoma within the spinal cord is relatively rare compared to that of extramedullary melanocytoma. A total of 29 cases of intramedullary meningeal melanocytoma were found in the public databases, PubMed and Google Scholar.

\section{Diagnosis}

The majority of patients from the reported cases were admitted to hospitals with symptoms of motor weakness, myelopathy, and/or sensory changes. Cinicians are favoring MRI in order to diagnose the disease. In common, intramedullary meningeal melanocytoma appears as iso-intensity to mild hyperintensity on TIW and iso-intensity to hypointensity on T2. Gadolinium contrast slightly enhances the lesion homogenously ${ }^{11}$. The specificity of MRI for the disease entity of melanocytoma is not sufficient to diagnose the disease alone. In our case, the impression from the MRI images was cavemous malformation, not melanocytoma.

$F^{18}$-FDG PET/CT is a great tool for the differential diagnosis of malignancy in addition to MRI. Primary melanoma and melanocytoma show similar features on MRI. However, biochemically, malignant tumors increase the activity of glucose transporter proteins and glucose phosphorylating hexokinase, which can be detected by $\mathrm{F}^{18}$-FDG $\mathrm{PET} / \mathrm{CT}^{12}$.

Pathologic confirmation is the most important step in diagnosing the disease. The key to diagnosis of the disease is associated with melanin in the cells. Melanocyitic tumors such as melanoma and melanocytoma are occasionally unpigmented. However, they can be diagnosed under an electron microscope or using immunohistochemical staining techniques. The tumors showed oval or spindle shape with melanin content. The distinct histological features are positive reactions to anti-melanoma antibody (HMB-45) and S-100 but negative for epithelial membrane antigen (EMA) ${ }^{7}$. Cellular morphology is considered to be a prognostic factor. The spindle-shaped morphology shows a relatively better prognosis than oval-shaped ${ }^{13}$. The difference between melanoma and melanocytoma is the degree of abnormal mitosis and atypical cytology. Melanocytoma generally shows an absence or a low-grade atypical cytology ${ }^{7}$. In our case, the tumor showed a spindle-shaped melanin content with a low grade of mitosis $(\mathrm{Ki}-67<5 \%)$ and no severe dysplasia under the microscope.

Immunohistochemical markers are not useful when it comes to distinguishing malanocytic tumors due to the almost identical reactivity to HMB-45 and S-100 14 . Molecular analyses have recently been highlighted to solve this problem. Melanocytoma shows no mutation in the $G$ alpha $q(G N A Q)$ gene on the long arm of chromosome 9, but melanoma does. The GNAQ gene encodes oncop- rotein that activates the mitogen activation protein (MAP) kinase pathway that is related to malignancy. Hoffmann et al. (2016) used molecular analyses of GNAQ and avoided the misdiagnosis of malignant melanoma instead of melanocytomas ${ }^{15}$. It is also useful to determine whether the lesion primarily originates from the CNS. GNAQ and guanine nucleotide-binding protein subunit alpha-11 (GNA17) gene mutations are distinct features of the CNS melanocytic tumors ${ }^{14}$. Molecular analyses were recommended to the patient for diagnosis with a cost, but the patient refused to do so because of the poor financial status.

\section{Treatment}

No consensus was made with the first-line treatment for the tumor. However, many case reports have recommended its complete resection. Melanocytoma is generally considered a benign disease but has the potential to recur and become malignant, 75,16 .

Adjuvant radiotherapy is also emphasized by many authors for the reasons mentioned above. Rades et al. (2004) reported a prognostic analysis of intracranial and intraspinal meningeal melanocytomas ${ }^{17}$. The patients who underwent complete resection showed $100 \% 5$-year sunvival rate s regardless of radiotherapy. However, incomplete resection showed a difference. The group receiving adjuvant radiotherapy showed almost 2-fold higher 5 -year survival rates than the group without radiotherapy ${ }^{17}$. Intramedullary meningeal melanocytoma might show a similar prognosis to that of other meningeal melanocytomas since the origin of tumor cells are identical.

\section{CONCLUSION}

Intramedullary meningeal melanocytoma in the cervical spine is an extremely rare tumor. Here, we reported the case with a narrative literature review. Magnetic resonance imaging (MRI) is the most frequently used diagnostic tool. However, the degree of sensitivity and specificity of MRI are insufficient to confim intramedullary meningeal melanocytoma. Microscopic confirmation using immunohistochemical markers is an essential step for the final diagnosis. For the treatment of diseases, the most important aspect of prognosis is the complete resection of intramedullary meningeal melanocytoma. In addition, adjuvant radiotherapy should be considered even though complete resection has been performed. The possibility of a remnant tumor on the resection margin always exists even if it has been completely removed. Hence, adjuvant radiotherapy should not be omiHed.

\section{REFERENCES}

1. Limas C, Tio FO: Meningeal melanocytoma ("melanotic meningioma"). Its melanocytic origin as revealed by electron microscopy. Cancer 30(5):1286-1294, 1972

2. Trinh V, Medina-Flores R, Taylor CL, Yonas H, Chohan MO: Primary melanocytic tumors of the central nervous system: Report of two cases and review of literature. Surg Neurol Int 5: 147, 2014

3. Cetinalp NE, Yildirim AE, Divanlioglu D, Belen D: An Uncommon Intramedullary Tumor: Primary Spinal Cord Melanoma. 
Asian Spine Journal 8(4):512-515, 2014

4. Francois P, Lioret E, Jan M: Primary spinal melanoma: Case report. Br J Neurosurg 12(2):179-182, 1998

5. Schoenwolf GC, Bleyl SB, Brauer PR, Francis-West PH: Larsen's human embryology. Fifth edition. ed. Philadelphia, PA: Churchill Livingstone; 2015

6. Dupin E, Le Douarin NM: Development of melanocyte precursors from the vertebrate neural crest. Oncogene 22(20):30163023, 2003

7. Liubinas SV, Maartens N, Drummond KJ: Primary melanocytic neoplasms of the central nervous system. J Clin Neurosci 17(10):1227-1232, 2010

8. Miler AJ, Martin C Mihm J: Melanoma. N Engl J Med 355: 51-65, 2006

9. Dubey A, Kataria R, Sardana VR: Intramedullary Melanocytoma of the Cervicothoracic Cord: Case Report and Review of Literature. Asian J Neurosurg 13(2):478-481, 2018

10. Karikari IO, Powers CJ, Bagley CA, Cummings TJ, Radhakrishnan S, Friedman AH: Primary intramedullary melanocytoma of the spinal cord: Case report. Neurosurgery 64(4):E777778; discussion E778, 2009

11. Merhemic Z, Stosic-Opincal T, Thurnher MM: Neuroimaging of Spinal Tumors. Magn Reson Imaging Clin N Am 24(3): 563579, 2016

12. Zhang M, Liu R, Xiang Y, et al.: Primary Spinal Cord Melanoma: A Case Report and a Systemic Review of Overall Survival. World Neurosurg 114:408-420, 2018

13. McLean IW, Saraiva VS, Burnier MN: Pathological and prognostic features of uveal melanomas. Canadian Journal of Oph thalmology 39(4):343-350, 2004

14. Narvaez-Martinez Y, Sagrista-Garcia M, Fernandez-Figueras MT: Letter to the Editor: Role of mutational status of GNAQ and GNA11 in the diagnosis of melanocytic tumors. J Neuro surg 126(3):1024-1026, 2017

15. Hoffmann M, Koelsche C, Seiz-Rosenhagen M, et al.: The GNAQ in the haystack: Intramedullary meningeal melanocytoma of intermediate grade at T9-10 in a 58-year-old woman. J Neurosurg 125(1):53-56, 2016

16. Rades D, Heidenreich F, Tatagiba M, Brandis A, Karstens JH: Therapeutic options for meningeal melanocytoma. Case report. J Neurosurg. 95(2 Suppl):225-231, 2001

17. Rades D, Schild SE, Tatagiba M, Molina HA, Alberti W: Therapy of meningeal melanocytomas. Cancer 100(11):2442-2447, 2004

18. Barth A, Pizzolato GP, Berney J: Intramedullary meningeal melanocytoma. Neurochirurgie 39(3):188-194, 1993

19. Glick R, Baker C, Husain S, Hays A, Hibshoosh H: Primary melanocytomas of the spinal cord: A report of seven cases. Clin Neuropathol 16(3):127-132, 1997

20. Delhaye M, Menei P, Rousselet MC, Diabira S, Mercier P: A case of intramedullary primary melanocytic tumor: Meningeal melanocytoma or malignant melanoma?. Neurochirurgie 47(2-3 Pt 1):133-136, 2001

21. Iida M, Llena JF, Suarez MA, et al.: Two cases of spinal meningeal melanocytoma. Brain Tumor Pathol 19(1):41-45, 2002

22. Turhan T, Oner K, Yurtseven T, Akalin T, Ovul I: Spinal meningeal melanocytoma. Report of two cases and review of the literature. J Neurosurg 100(3 Suppl Spine):287-290, 2004

23. Van Paesschen R VCF, Demaerel P, Sciot T: Intramedullary melanocyte a associated with syringobulbia. Acta Neurologica Belgica 104:132-133, 2004

24. Horn EM, Nakaji P, Coons SW, Dickman CA: Surgical treatment for intramedullary spinal cord melanocytomas. J Neurosurg Spine 9(1):48-54, 2008

25. Chacko G, Rajshekhar V: Thoracic intramedullary melanocytoma with long-term follow-up. J Neurosurg Spine 9(6):589592, 2008

26. Caruso R, Marrocco L, Wierzbicki V, Salvati M: Intramedullary melanocytoma: Case report and review of literature. Tumori 95(3):389-393, 2009

27. Perrini P, Caniglia M, Pieroni M, Castagna M, Parenti GF: Malignant transformation of intramedullary melanocytoma: Case report. Neurosurgery 67(3):E867-869; discussion E869, 2010

28. Eskandari R, Schmidt MH: Intramedullary spinal melanocytoma. Rare Tumors 2(2):e24, 2010

29. Muthappan M, Muthu T, Hussain Z, Lamont D, Balakrishnan V: Cervical intramedullary melanocytoma: A case report and review of literature. J Clin Neurosci 19(10):1450-1453, 2012

30. Kahilogullari G: Primary Intramedullary Melanocytoma: A Case Report. Journal of Clinical Case Reports 02(06), 2012

31. Wagner F, Berezowska S, Wiest R, et al.: Primary intramedullary melanocytoma in the cervical spinal cord: Case report and literature review. Radiol Case Rep 10(1):1010, 2015

32. Reutov AA, Ryzhova MV, Kushel YV: Intramedullary melanocytoma: A clinical case report and literature review. $\mathrm{Zh}$ Vopr Neirokhir Im N N Burdenko 80(4):75-80, 2016

33. Khoulali M, Haouas MY, Mortada J, Srour R: Meningeal melanocytoma: aggressive evolution of a benign tumor: About 2 cases. Pan Afr Med J 29:211, 2018

33. Van Paesschen R, Van Calenbergh F, Demaerel P, Sciot T: Intramedullary melanocyte a associated with syringobulbia. Acta Neurologica Belgica 104:132-133, 2004 